\title{
EDITORIAL
}

\section{Obsessive-compulsive and related disorders in ICD-11}

\author{
Dan J. Stein, ${ }^{1}$ Leonardo F. Fontenelle, ${ }^{2,3,4}$ Geoffrey M. Reed ${ }^{5}$ \\ ${ }^{1}$ Department of Psychiatry, University of Cape Town, Groote Schuur Hospital, Cape Town, South Africa. ${ }^{2}$ Institute of Psychiatry, Universidade \\ Federal do Rio de Janeiro (UFRJ), Rio de Janeiro, RJ, Brazil. ${ }^{3}$ Instituto D'Or de Pesquisa e Ensino, Rio de Janeiro, RJ, Brazil. ${ }^{4}$ School of \\ Psychological Sciences, Monash University, Melbourne, Australia. ${ }^{5}$ Department of Mental Health and Substance Abuse, World Health \\ Organization, Geneva, Switzerland.
}

Considerable attention has been devoted in recent years to the issue of whether there is a "spectrum" of obsessive-compulsive and related disorders (OCRD). 1,2 The core features of this grouping of disorders, which potentially includes obsessive-compulsive disorder (OCD), body dysmorphic disorder, and hypochondriasis, are intrusive and unwanted thoughts or preoccupations and related repetitive behaviors, including mental rituals. Such conditions may not only have some phenomenological similarities, but also share some psychobiological features. Evidence for the validity of the grouping includes a high degree of familiality, shared genetic liability and neurocircuitry, and similar treatment responsiveness. Hoarding disorder, being considered as a new entry in ICD-11, is also characterized by repetitive urges and behaviors related to acquisition, and has been historically associated with OCD. Other conditions, such as trichotillomania, skin-picking disorder, and Tourette's disorder, are characterized by body-focused repetitive behaviors and seem to be more common in family members of OCD probands. Although there continues to be controversy related to the splitting of some of these disorders particularly OCD and hypochondriasis - from the anxiety disorders, their grouping in a separate cluster may be clinically useful because of high rates of comorbidity among them and the similarity of effective treatment approaches. DSM $-5^{3}$ included a new grouping of OCRD, recognizing its clinical utility and diagnostic validity.

The ICD-11 Working Group on Obsessive-Compulsive and Related Disorders was asked to consider evidence regarding the validity of this grouping of disorders and its constituent members, as well as its clinical utility. The Working Group was also asked to consider the proposals that had been made for DSM-5 (at that time, prior to its publication) and their potential global applicability. Around the world, most individuals with mental disorders continue to be seen in primary care settings, by non-specialist clinicians, and a grouping of OCRD would also be new to many specialist health professionals, so it was important to develop a compelling clinical rationale to help them use it appropriately. ${ }^{4}$ The manuscripts in this special issue of the Revista Brasileira de Psiquiatria provide the results of the Working Group's examination of each relevant diagnostic category, including an examination of their history in both the ICD and the DSM, and options and recommendations for ICD-11. The World Health Organization is particularly concerned with the utility of the ICD-11 in low- and middle-income countries, where $85 \%$ of the world's people live. ${ }^{5}$ The Revista Brasileira de Psiquiatria represents a particularly important psychiatric journal in the Global South, so the Working Group is especially pleased to publish its reviews in this issue.

One notable recommendation that can be taken from the articles in this issue is that the ICD-11 chapter on OCRD be somewhat broader than that in DSM-5. Olfactory reference syndrome, for example, has been reported around the world, and appears to have much in common with body dysmorphic disorder; the Working Group has therefore recommended its inclusion in the ICD-11, as described by Veale \& Matsunaga. While DSM-5 retains illness anxiety disorder in the section on somatic symptom disorders, van den Heuvel et al. note that hypochondriasis has elements in common with both the obsessivecompulsive grouping and with anxiety disorders. Finally, while DSM-5 includes Tourette syndrome in the section on neurodevelopmental disorders, ICD-11 will include this in the chapter on Diseases of the Nervous System. Woods \& Thomsen point out that there is clinical utility in crossreferencing the condition in the section on OCRD. At the same time, the Working Group has not recommended that the construct of OCRD be extended to include a range of impulse control disorders, but rather has recommended that pathological gambling and compulsive sexual behavior disorder be classified in the ICD-11 section on impulse control disorders, which also includes pyromania, kleptomania, and intermittent explosive disorder. ${ }^{6}$

Given the multiple mechanisms that contribute to mental disorders and the heterogeneity of any particular condition, it would be surprising if all of psychopathology could be neatly captured in a single dimensional space. ${ }^{7}$ The notion of cross-referencing certain disorders in different parts of the nomenclature emphasizes that particular conditions can be viewed equally appropriately from different vantage points. For example, on the one hand, it is important for neurologists to conceptualize tic disorders as one of many conditions characterized by involuntary movements. On the other hand, it is important for clinicians to remember to assess individuals with OCRD for tics, as patients with and without tics have important clinical differences, and may require somewhat different treatment approaches.

The Working Group welcomes feedback from the field on the proposals it has developed and presents in this issue. There is growing awareness of the importance 
of including patient perspectives, and these too have influenced the construct of OCRD. Furthermore, we will be testing the diagnostic guidelines based on the work described here in internet-based field studies using the Global Clinical Practice Network, a global network currently consisting of nearly 11,000 individual mental health and primary care professionals in more than 130 countries (www.globalclinicalpractice.net), as well as in clinic-based field studies using WHO's network of International Field Study Centers. Ultimately, we hope that the proposals put forward by the WHO ICD-11 Working Group on Obsessive-Compulsive and Related Disorders will contribute to the improved clinical utility of ICD-11.

\section{Acknowledgements}

The Department of Mental Health and Substance Abuse, World Health Organization, has received direct support that contributed to the activities of the Working Group from several sources: the International Union of Psychological Science, the National Institute of Mental Health (USA), the World Psychiatric Association, the Royal College of Psychiatry (United Kingdom), the Spanish Foundation of Psychiatry and Mental Health (Spain), and the Santander Bank UAM/UNAM endowed Chair for Psychiatry (Spain/ Mexico).

DJS is supported by the Medical Research Council of South Africa. LFF is supported by Conselho Nacional de Desenvolvimento Científico e Tecnológico (CNPq) and Fundação de Amparo à Pesquisa do Estado do Rio de Janeiro (FAPERJ).

\section{Disclosure}

DJS and LFF are members of the WHO ICD-11 Working Group on the Classification of Obsessive-Compulsive and Related Disorders, reporting to the International Advisory Group for the Revision of ICD-10 Mental and Behavioural Disorders. GMR is a member of the WHO Secretariat responsible for coordinating the activities of the Working Group. Unless specifically stated, the views expressed in this editorial are those of the authors and do not represent the official policies or positions of the Working Group, of the International Advisory Group, or of the WHO. DJS has received research grants and/or consultancy honoraria from AMBRF, Biocodex, Cipla, Lundbeck, the National Responsible Gambling Foundation, Novartis, Servier, and Sun. The authors report no other conflicts of interest.

\section{References}

1 Phillips KA, Stein DJ, Rauch SL, Hollander E, Fallon BA, Barsky A, et al. Should an obsessive-compulsive spectrum grouping of disorders be included in DSM-V? Depress Anxiety. 2010;27:528-55.

2 Storch EA, Abramowitz J, Goodman WK. Where does obsessivecompulsive disorder belong in DSM-V? Depress Anxiety. 2008; 25:336-47.

3 American Psychiatric Association. Diagnostic and Statistical Manual of Mental Disorders, Fifth Edition (DSM-5). Arlington: American Psychiatric Publishing; 2013.

4 Reed GM, Roberts MC, Keeley J, Hooppell C, Matsumoto C, Sharan $P$, et al. Mental health professionals' natural taxonomies of mental disorders: implications for the clinical utility of the ICD-11 and the DSM-5. J Clin Psychol. 2013;69:1191-212.

5 International Advisory Group for the Revision of ICD-10 Mental and Behavioural Disorders. A conceptual framework for the revision of the ICD-10 classification of mental and behavioural disorders. World Psychiatry. 2011;10:86-92.

6 Grant JE, Atmaca M, Fineberg NA, Fontenelle LF, Matsunaga H, Janardhan Reddy $\mathrm{YC}$, et al. Impulse control disorders and "behavioural addictions" in the ICD-11. World Psychiatry. 2014;13:125-7.

7 Stein DJ. Is disorder $X$ in category or spectrum Y? General considerations and application to the relationship between obsessivecompulsive disorder and anxiety disorders. Depress Anxiety. 2008;25:330-5. 